African Crop Science Journal by African Crop Science Society is licensed under a Creative Commons Attribution 3.0 Uganda License. Based on a work at www.ajol.info/ and www.bioline.org.br/cs DOI: https://dx.doi.org/10.4314/acsj.v27i2.2

\title{
FERTILISATION DES SOLS AVEC LA LITIERE FOLIAIRE EN ZONE SUBSAHELIENNE DU BURKINA FASO : DIVERSITE DES ESPECES, EFFETS SUR LES RENDEMENTS DU SORGHO
}

\section{BAMBARA, H. COMPAORE, S. SOULAMA ${ }^{1}$, Y. SAMANDOULGOU² et A. BILGO}

Centre National de la Recherche Scientifique et Technologique / Institut de l'Environnement et de Recherches Agricoles (CNRST/INERA), Département de Gestion des Ressources Naturelles et Systèmes de Productions (GRN-SP), 01 BP 476, Ouagadougou 01, Burkina Faso

${ }^{1}$ Centre National de la Recherche Scientifique et Technologique / Institut de l'Environnement et de Recherches Agricoles (CNRST/INERA), Département Environnement et Forêts (DEF), 04 BP 8645, Ouagadougou 04, Burkina Faso

${ }^{2}$ Centre National de la Recherche Scientifique et Technologique / Institut de l'Environnement et de Recherches Agricoles (CNRST/INERA), Centre Régional de Recherches Environnementales et Agricoles (CRREA) du Centre, BP 10, Saria, Koudougou, Burkina Faso

Auteur correspondant : das.bambara@coraf.org, bambaradasmane@yahoo.fr

(Received 27 February 2019; accepted 16 April 2019)

\section{RÉSUMÉ}

La gestion des sources de matières organiques, pour améliorer la fertilité des sols, a fait l'objet de nombreuses recherches. L'agroforesterie est une des thématiques abordées par ces recherches. Dans le Centre-Nord du Burkina Faso, la litière foliaire des espèces ligneuses est ramassée, mise en tas, émiettée avant d'être épandue dans les champs. Cette pratique est peu connue dans le reste du pays. La présente étude visait à fournir des informations sur la diversité des espèces végétales spontanées concernées, les motivations paysannes de l'émiettement de la litière et à évaluer, empiriquement et par des mesures, les effets de l'épandage de ces litières sur les rendements du sorgho. Une étude participative, à l'aide d'enquêtes qualitatives et des mesures biométriques dans des champs, a été menée dans cinq villages du Centre Nord du Burkina Faso. A cause de leur disponibilité, leur accessibilité, leur performance agronomique, les plantes telles que Vitellaria paradoxa, Anogeissus leiocarpa, Combretum micranthum, Lannea microcarpa, Diospyros mespiliformis ont été citées comme étant les espèces dont la litière des feuilles est valorisée. Les mesures réalisées in situ montrent que, les rendements du sorgho dans les champs ayant reçu de la litière foliaire de Vitellaria pour la première fois, ont été inférieurs à ceux des parcelles témoin. Par contre, les champs ayant reçu cette litière pendant trois années consécutives ont été plus productifs. Dans l'optique de valider les savoirs locaux et d'approfondir les connaissances sur l'effet des litières foliaires sur la fertilité du sol, une étude scientifique sur leur caractéristique chimique et sur leur influence sur les paramètres de fertilité du sol, est à envisager.

Mots Clés : Agroforesterie, Burkina Faso, pratiques locales, matière organique, sondage 


\begin{abstract}
Organic matter sources management for soil fertility improvement has been the subject of many researches. Agroforestry is one of the topics addressed by these researches. In Burkina Faso's Northern Center, leaf litter of woody species is collected, piled, crumbled before spreading in fields. This practice is not well known in the rest of the country. The present study aims to provide information on the diversity of spontaneous plant species concerned, farmers' motivations of litter crumbling and to evaluate, empirically the effects of litter spreading on sorghum yields. A participatory study, using qualitative surveys and biometric field measurements, was conducted in five villages in the Northern Center of Burkina Faso. Because of their availability, accessibility, and agronomic performance, plants such as Vitellaria paradoxa, Anogeissus leiocarpa, Combretum micranthum, Lannea microcarpa and Diospyros mespiliformis were the species whose leaf litter was valued most. Measurements achieved in situ indicate that sorghum yields from fields that received Vitellaria leaf litter for the first time were lower than those in the control plots. On the other hand, fields that received this litter for three consecutive years were more productive. A scientific study on leaf litter chemical characteristics and their influence on soil fertility parameters should be considered in order to validate local knowledge and to improve knowledge about these practices.
\end{abstract}

Key Words: Agroforestry, Burkina Faso, local practices, organic matter, survey

\section{INTRODUCTION}

Au Burkina Faso, et en particulier dans la zone soudano-sahélienne, la majorité de la population vit d'une agriculture utilisant peu d'intrants (Hien et al., 2010). Traditionnellement, cette méthode d'agriculture s'appuyait sur la jachère pour entretenir la fertilité du sol. Mais, les changements climatiques combinés à l'augmentation de la densité de la population ont entraîné le raccourcissement de la durée, voire la disparition de la jachère (Floret et Pontannier, 1997). Cette situation s'est accompagnée d'une diminution de la fertilité et particulièrement l'appauvrissement du sol en matière organique, facteur principal responsable de sa fertilité dans ces milieux (Jouve, 2010).

Les ressources organiques jadis utilisées pour maintenir la fertilité organique (résidus de culture notamment) ne sont plus disponibles du fait d'une forte compétition (Bambara et al., 2017). D'autres facteurs naturels (topographie, caractéristiques physicochimiques des sols) et techniques (système de production) contribuent à la dégradation progressive des ressources en sols de la zone. Il convient donc de prendre en compte et de gérer au mieux toutes les ressources organiques au niveau des terroirs, dans une optique de gestion durable de la qualité des sols.

Plusieurs travaux relatifs à la pratique de l'agroforesterie, comme moyen d'améliorer la fertilité des sols, ont été menés au Burkina Faso (Bayala et al., 2006 ; Bationo et al., 2012) et dans sa partie centrale en particulier (Yaméogo et al., 2005 ; Yélémou et al., 2007). Ces travaux ont montré l'importance de l'arbre dans le maintien de la fertilité organique des sols via la chute les feuilles. Ces feuilles constituent, en effet, une ressource organique relativement disponible, susceptible d'amender les sols (Yélémou et al., 2013).

Dans la province du Sanmatenga, dans le Centre-Nord du Burkina Faso, la litière foliaire des ligneux est traitée de façon spéciale avant d'être apportée dans les champs comme source de fertilisants. En effet, à la différence de la valorisation des feuilles des ligneux dans le système agroforestier traditionnel (où les feuilles qui chutent sont laissées en l'état), dans cette zone, la litière foliaire est ramassée, mise en tas, broyée dans des sacs avant d'être 
épandue dans les champs. Cette pratique, presque inconnue dans le reste du pays, malgré quelques travaux y relatifs (Kabré, 2010 ; Ouédraogo, 2014), n'est pas documentée et les effets de la litière ainsi traitée sur les cultures restent peu connus. L'objectif de l'étude était de déterminer la diversité des espèces végétales concernées et d'évaluer les effets des litières prétraitées sur le développement du sorgho, principale culture vivrière dans la zone.

\section{MATÉRIELS ET MÉTHODES}

Sites d'étude. L'étude a été conduite au Burkina Faso dans les villages de Louda, Boré, Nakourtenga, Saab-Toécé et Santèna, dans la Province du Sanmatenga, Centre-Nord du pays. La zone appartient au secteur phytogéographique subsahélien (Fontès et Guinko, 1995) (Fig. 1). La pluviosité annuelle moyenne varie de 500 à $600 \mathrm{~mm}$. Les températures moyennes oscillent entre un minimum de $17,7^{\circ} \mathrm{C}$ pendant les mois de décembre et de janvier et un maximum de $40^{\circ} \mathrm{C}$ au cours des mois de mars et d'avril. Les valeurs de l'évapotranspiration potentielle (ETP) restent élevées avec un cumul de plus de $2 \mathrm{~m}$ d'eau en moyenne par an (SP/ CONEDD, 2006).

Les types de sols dominants sont les sols peu évolués d'érosion gravillonnaire, les sols ferrugineux tropicaux lessivés et les lithosols sur cuirasse (BUNASOLS, 1987). L'environnement écologique se caractérise par une végétation qui se partage entre savane (arborée à épineux divers) et steppe dans un cadre pédologique relativement dégradé (Fontès et Guinko, 1995).

Les populations dans la région de cette étude pratiquent dans leur majorité une agriculture extensive de subsistance familiale (MEF, 2010). Les feux de brousses, la monoculture céréalière continue sans jachère, l'érosion hydrique et le surpâturage sont les principaux

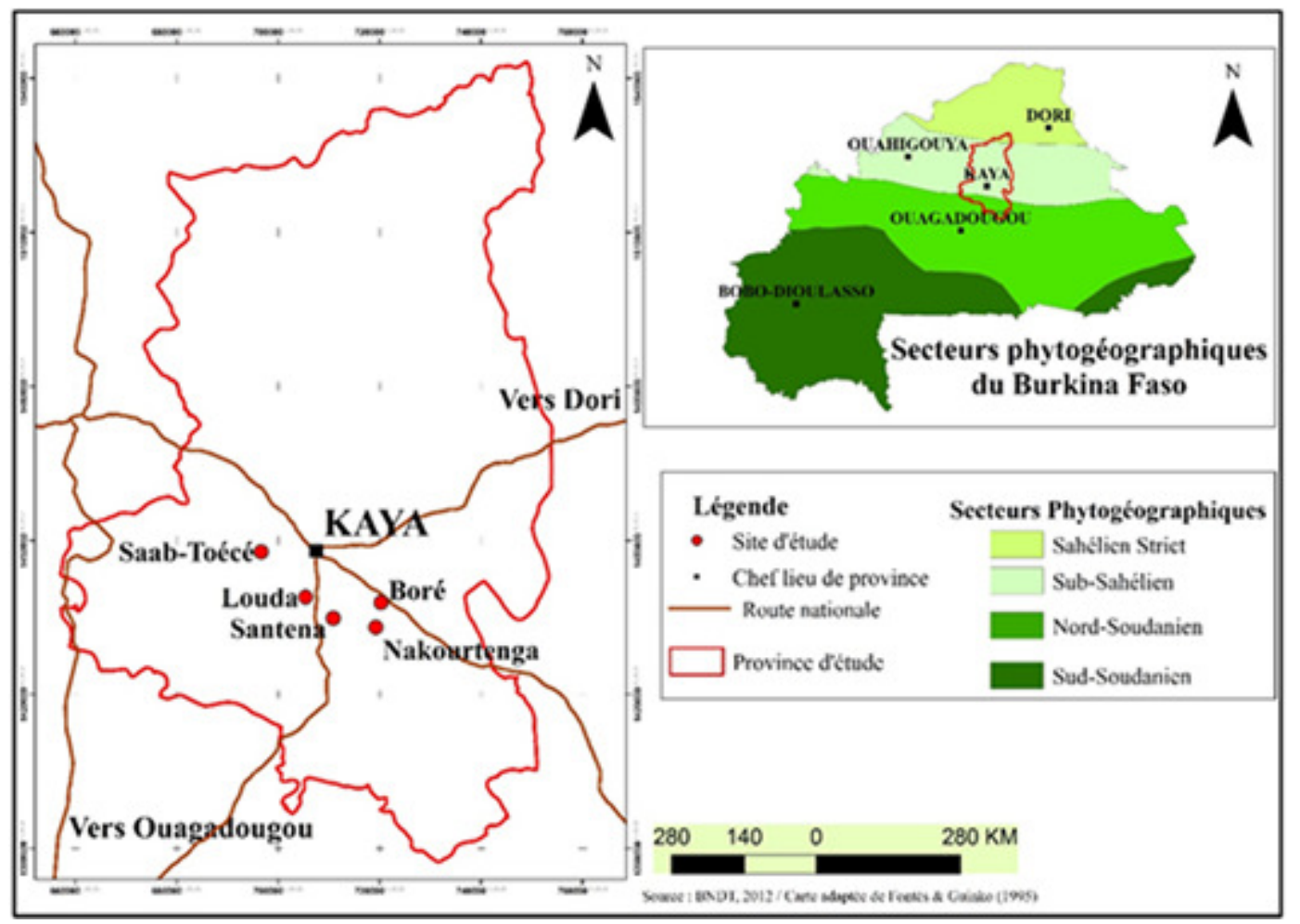

Figure 1. Localisation des sites d'étude dans la Province du Sanmatenga. 
facteurs anthropiques et environnementaux à l'origine de la perte de productivité des sols. Les cinq villages-sites ont été choisis à cause de leur accessibilité en toute saison et de la volonté de leur population à collaborer.

Collecte des données. Deux méthodes de collecte de données ont été utilisées : les enquêtes-observations et les mesures biométriques dans les champs de paysans.

Enquêtes-observations. Il s'était agi d'enquêtes qualitatives informelles menées dans chaque village. Elles ont consisté en des entretiens de groupes non-directifs au cours desquels les enquêtés étaient pleinement libres des réponses qu'ils apportaient aux questions relatives au thème qui leur était proposé. Selon Magioglou (2008), ce type d'entretien est intéressant lorsqu'il s'agit de recueillir des perceptions, des expériences ou des attitudes par rapport à un phénomène donné.

Les entretiens ont été réalisés avec les producteurs au cours de rencontres villageoises (Tableau 1) facilitées par les acteurs locaux de développement. Les questions essentielles suivantes avaient été posées : (i) «Quelles sont les plantes ligneuses sauvages dont vous utilisez les feuilles pour fertiliser vos champs?»; (ii) «Quelles sont les espèces dont les litières sont plus fréquemment utilisées ? » et (iii) «Comment et pourquoi prétraitez-vous les litières foliaires avant leur application dans les champs, comment appréciez-vous les effets des litières ainsi traitées sur les rendements du sorgho ?».

TABLEAU 1. Effectifs genres des participants aux enquêtes dans les villages

\begin{tabular}{lccc}
\hline Villages & Hommes & Femmes & Total \\
\hline Louda & 38 & 23 & 61 \\
Boré & 25 & 15 & 40 \\
Nakourtenga & 41 & 28 & 69 \\
Saab-Toécé & 32 & 25 & 57 \\
Santèna & 39 & 25 & 64 \\
\hline
\end{tabular}

Mesures biométriques. Pour des raisons de contraintes de terrain, les mesures biométriques ont été effectuées dans des champs ayant reçu de la litière foliaire prétraitée de Vitellaria paradoxa. Il s'est agi de la hauteur à maturité et des rendements en grain et en paille du sorgho. Pour ce faire, dans le village de Louda, trois champs, contigus, de superficies inférieures à un hectare et ayant été traités différemment avec la litière ont été retenus pour les mesures. Le premier champ a reçu la litière pour la première fois en 2018, ce champ a été considéré comme la première parcelle. Le deuxième champ a reçu la litière sur trois années consécutives : 2016, 2017 et 2018, ce champ a été considéré comme la seconde parcelle. Le troisième champ n'a jamais reçu de la litière, ce champ a été considéré comme la parcelle témoin (contrôle). Compte tenu de la taille relativement réduite des parcelles ( $<1 \mathrm{ha})$, trois carrés de rendement (placettes de $25 \mathrm{~m}^{2}$ ) ont été posés de façon aléatoire à l'intérieur de chaque parcelle à l' aide du « demi carré » de $5 \mathrm{~m}$ de côté (Bambara et al., 2017). Chaque placette, considérée comme une répétition, est matérialisée par quatre piquets en bois et délimitée par une ficelle en matière plastique de couleur jaune. C'est à l'intérieur de ces placettes que les mesures de hauteur et de rendements ont été réalisées.

Mesure de hauteur. La mesure de hauteur a été effectuée après exertion complète de la feuille paniculaire, moment où la croissance en hauteur du sorgho est terminée (Reddy et al., 1998). La hauteur de la tige est prise du collet au sommet de la panicule (Reddy et al., 1998). La mesure a concerné les plantes de 10 poquets pris au hasard dans chaque placette. Le choix des pieds à mesurer a été réalisé de façon aléatoire par un tirage au sort (Bambara et al., 2017). La moyenne des hauteurs, dans les trois placettes de chaque parcelle, constituait la hauteur du sorgho dans la parcelle (traitement) considérée.

A maturité (110 jours après semis), les panicules et la paille du sorgho de chaque placette de rendement ont été récoltées 
séparément et séchées au soleil jusqu'à l'obtention de poids secs constants. Les panicules ont été battues puis vannées pour obtenir les grains. Les grains ont été pesés avec une balance à ressort de marque HOMS de 1 $\mathrm{kg}$ de portée $\pm 10 \mathrm{~g}$. La paille, mise en bottes, a été pesée à l'aide d'une balance à ressort de marque HOMS de $5 \mathrm{~kg}$ de portée avec une précision de $50 \mathrm{~g}$ pour déterminer la biomasse paille. Les poids obtenus ont été extrapolés à l'hectare pour obtenir les rendements.

Analyse des données. Les données issues des enquêtes ont été saisies et traitées à l'aide du logiciel SPSS (Statistical Package for the Social Sciences)11.0 (Norusis, 2002). L'effectif des producteurs ayant cité et l'effectif des producteurs ayant adopté les espèces végétales dont les litières foliaires sont valorisées ont été traduits sous forme de proportions centésimales. Les données des mesures de hauteur et de rendements en biomasses grain et paille du sorgho ont été analysées avec le logiciel XLSTAT Version 20.7.55194 (Addinsoft, 2018). Les procédures statistiques ont été l'analyse de variance ANOVA à un facteur et la séparation des moyennes a été faite par le test de Fischer au seuil de 5\%. Les comparaisons ont été faites entre les trois parcelles différemment traitées avec la litière de Vitellaria paradoxa. La relation entre les rendements en biomasses grain/paille et la hauteur du sorgho à maturité a été établie par tracé graphique. Les équations de régression ont été déterminées par le test de corrélation de Pearson au seuil de signification de 5\%. Le tracé des graphiques a été réalisé avec le même logiciel.

\section{RÉSULTATS ET DISCUSSION}

Espèces utilisées. L'inventaire des plantes ligneuses dont la litière foliaire est valorisée dans les champs par les producteurs a permis de recenser cinq espèces végétales appartenant à cinq genres et trois familles (Tableau 2).

Seule la famille des combretaceae était représentée par deux espèces ; les autres familles étant représentées par une seule espèce. A l'exception de Diospyros mespiliformis dont la frondaison était persistante, les autres espèces étaient caducifoliées. La chute des feuilles intervenait pendant la saison sèche, de décembre de l'année $n$, à avril de l'année $n+1$. Il ressort une faible diversité des espèces utilisées par les enquêtés, puisque Belem et al. (2008) ont indiqué que, dans cette zone, il existerait plus de 29 espèces agroforestières. En effet, des espèces telles que Parkia biglobosa (Jacq.) Benth., Adansonia digitata L., Faidherbia albida (Del.) A. Chev., Piliostigma reticulatum (DC) Hochst., Bombax costatum Pellegr. et Vuillet, Guiera senegalensis J.F. Gmel., Hyphaene thebaica (L.) Mart., Cajanus cajan (L.) Millsp., Gliricidia sepium (Jacq.) Kunth ex Walp., etc. ont été rapportées comme étant des espèces agroforestières (Bayala et al., 2006 ; Yélémou et al., 2007 ; Bationo et al., 2012). Cette faible diversité des espèces valorisées par les enquêtés est encore plus remarquable si on la compare aux potentialités

TABLEAU 2. Liste des espèces ligneuses par genre et famille

\begin{tabular}{lll}
\hline Familles & Genres & Espèces \\
\hline Sapotaceae & Vitellaria & Vitellaria paradoxa C.F. Gaertn. \\
Combretaceae & Anogeissus & Anogeissus leiocarpa (DC.) Guill. \& Perr. \\
Ebenaceae & Diospyros & Diospyros mespiliformis Hochst. ex A. DC. \\
Combretaceae & Combretum & Combretum micranthum G. Don \\
Anacardiaceae & Lannea & Lannea microcarpa Engl. \& K. Krause \\
\hline
\end{tabular}


du pays où 106 espèces agroforestières sont rapportées (Cissé et al., 2018). Ce constat s'expliquerait, non seulement, par l'adversité du milieu du fait de sa localisation à la lisière du sahel où la biodiversité est réduite (Fontès et Guinko, 1995), mais aussi par le fait de sa perpétuelle dégradation biophysique (FAO, 2010). Elle pourrait aussi être liée à des raisons culturelles car, la préservation des espèces végétales lors des défrichements varie selon le groupe ethnique, les traditions coutumières et les pratiques endogènes empiriques en présence (Ganaba et al., 1998 ; Traoré et al., 2011 ; Cissé et al., 2018).

Tous les producteurs enquêtés utilisent la litière foliaire de Vitellaria paradoxa pour fertiliser leurs champs. La litière foliaire de Combretum micranthum est utilisée par $37 \%$ des producteurs alors que celles de Anogeissus leiocarpa, Diospyros mespiliformis et Lannea microcarpa sont adoptées par moins de 16\% des enquêtés (Tableau 3). Cette différence, dans l'adoption des litières foliaires par les paysans, est sans doute liée à la disponibilité, à l'accessibilité et à l'importance socioéconomique des espèces sur le terrain. En effet, Vitellaria paradoxa est une espèce omniprésente dans les champs et les jachères environnantes. C'est une espèce qui a un intérêt socioéconomique connu dans le monde (Belem et al., 2008). Par ailleurs, ces feuilles chutent dans un rayon assez réduit qui facilite le ramassage, c'est ce qui justifierait son adoption par la quasi-totalité des enquêtés. Cissé et al. (2018) ont rapporté une adoption similaire pour cette espèce dans une étude sur la perception paysanne d'espèces agroforestières à Boura, zone soudanienne du Burkina Faso.

Cette espèce est suivie, en termes d'adoption, par Combretum micranthum, arbuste de taille réduite mais à feuillage abondant qui tombe aux alentours immédiats du tronc multicaule. Plante médicinale (Bognounou, 1987 ; Belem et al., 2008), elle est abondante dans les jachères situées à la lisière des champs. Mais sa rareté à l'intérieur des champs (espèce non conservée lors des défrichements) fait que son adoption par les paysans n'a pas été aussi importante, probablement à cause de la pénibilité du travail de ramassage et de transport de la litière.

Anogeissus leiocarpa, Diospyros mespiliformis et Lannea microcarpa ont été les espèces les moins adoptées par les enquêtés. Bien que faiblement représenté dans la zone, Anogeissus leiocarpa, avec 15,6\% d'adoption vient en troisième position. Cette espèce arborescente à feuilles de petite taille comparativement aux feuilles de Vitellaria paradoxa est, selon les paysans, l'espèce dont la litière foliaire améliorerait le mieux la qualité agronomique des sols.

A cause de cette dimension réduite des feuilles, les producteurs ne réalisent aucun prétraitement de sa litière avant son épandage dans les champs; l'effort physique lié à la valorisation de la litière est réduit, cela stimulerait son adoption. C'est une espèce endémique des formations steppiques

TABLEAU 3. Fréquences d'adoption (\%) de la litière foliaire par espèce et par village par les enquêtés

\begin{tabular}{lrrrrrr}
\hline Espèces & \multicolumn{4}{c}{ Fréquences d'adoption } & Moyennes \\
\cline { 2 - 6 } & Louda & Boré & Nakourtenga & Saab-Toécé & Santèna & \\
\hline Vitellaria paradoxa & 100 & 100 & 100 & 100 & 100 & 100 \\
Anogeissus leiocarpa & 13 & 17 & 30 & 8 & 10 & 15,6 \\
Diospyros mespiliformis & 21 & 8 & 15 & 9 & 5 & 11,6 \\
Combretum micranthum & 45 & 35 & 25 & 50 & 30 & 37 \\
Lannea microcarpa & 8 & 14 & 22 & 7 & 16 & 13,4 \\
\hline
\end{tabular}


sillonnées de minces forêts claires (surtout en zones riveraines) (Kessler et Boni, 1991). Lannea microcarpa, adopté avec un taux de $13,4 \%$ a été l'avant dernière espèce ciblée par les paysans. Malgré son intérêt socioéconomique (Belem et al., 2008), le faible engouement manifesté pour cette espèce serait lié à sa faible représentativité dans cet espace écologique subsahélien. Cette espèce est essentiellement endémique de la zone écologique soudano-sahélienne (Lebrun et al., 1991). Une fréquence de conservation de $30 \%$, dans les champs pour cette espèce, a été rapportée par Cissé et al. (2018) chez les Mossis dans l'extrême Sud du Burkina Faso.

La dernière espèce a été Diospyros mespiliformis avec seulement $11,6 \%$ de taux d'adoption. L'espèce est endémique des zones sud sahélienne et soudanienne (Lebrun et al., 1991). Les feuilles de cette espèce tombent très peu et la litière est donc peu disponible, c'est ce qui expliquerait sa faible adoption. Cissé et al. (2018) ont trouvé, chez les Mossis, une fréquence de présence dans les champs de moins de $15 \%$ pour cette espèce en zone soudanienne du Burkina Faso.

Techniques et motivations paysannes du prétraitement de la litière foliaire. Les populations rassemblent et mettent en tas la litière foliaire des ligneux à l'aide de branches d'arbustes ou de râteaux (Fig. 2). Le ramassage des feuilles est une activité généralement réservée aux femmes et aux enfants. Mais certains hommes, chefs de ménages ou jeunes participent activement aux activités. La litière ainsi amassée est mise dans des bâches localement confectionnées pour être émiettée par battage à l'aide de branches d'arbres ou par piétinement avant d'être épandue en tas dans les champs au cours du mois de mai (Fig. 2). Le ramassage a lieu durant les mois de février à avril. Durant cette période, l'harmattan souffle au Burkina Faso, et une des raisons de l'émiettement de la litière serait d'empêcher son transport par le vent car selon eux, l'émiettement permettrait une meilleure adhérence de la litière au sol. En outre, il faciliterait l'enfouissement de la litière dans le sol lors des opérations de scarifiages ou de labours, au cours de la préparation des lits de semis. Mais, la motivation principale évoquée par les producteurs serait d'accélérer le processus de décomposition de la litière et, partant, de son incorporation au sol. L'activité prend fin autour du mois de mai et ce n'est qu'à la prochaine saison qu'elle sera reconduite. Après l'épandage en tas de la litière dans les champs, son étalage sur les parties du champ à fertiliser n'interviendra qu'après les premières pluies, généralement au cours de la première quinzaine du mois de juin.

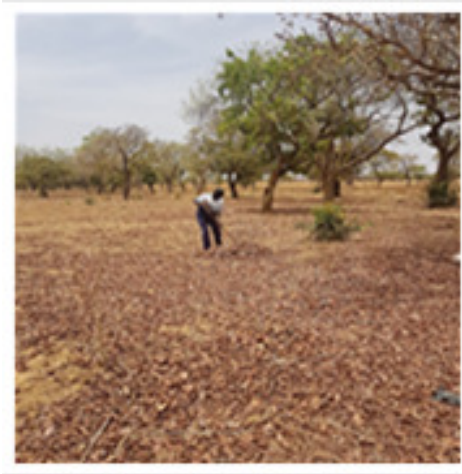

Ramassage-assemblage

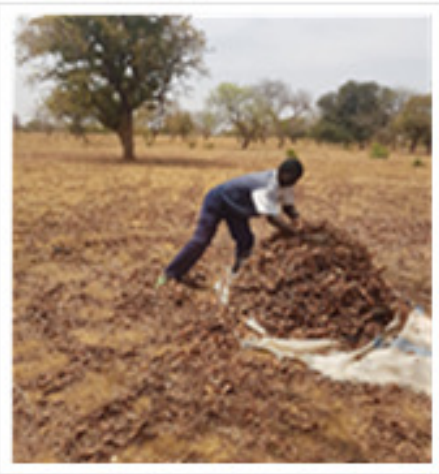

Mise en tas sur une bâche pour broyage (émiettement)

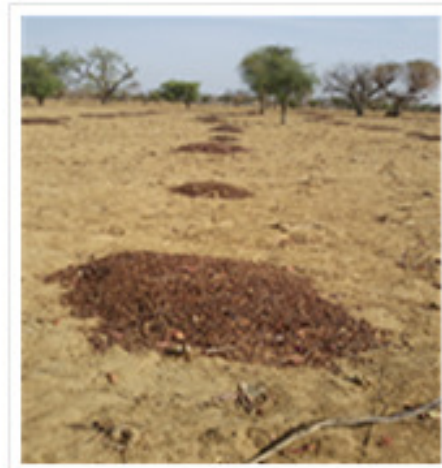

Epandage en tas de la litière émiettée

Figure 2. Illustrations du mode de gestion de la litière foliaire dans les champs. 
Perception endogène de l'effet des litières sur le rendement du sorgho. Les perceptions des paysans sur les effets des différentes litières foliaires sur le rendement du sorgho ont varié d'un village à l'autre selon les espèces (Tableau 4). Anogeissus leiocarpa, avec une fréquence de citation moyenne (tout village considéré) de $82,8 \%$, est l'espèce la plus perçue comme étant celle qui améliore le mieux le rendement du sorgho. Il est suivi par Combretum micranthum avec une fréquence de citation moyenne de $75,6 \%$, Lannea microcarpa avec 70,8\%, Diospyros mespiliformis avec un effet favorable sur les rendements perçu par $63,2 \%$ des enquêtés.

Par contre, Vitellaria paradoxa qui a été l'espèce dont la litière foliaire est adoptée par tous les enquêtés dans tous les villages, a été perçue comme améliorant les rendements par seulement $59,2 \%$ de tous les enquêtés des cinq villages. Son taux moyen de perception a été le plus bas. Ce constat amène à penser que l'adoption de la litière de Vitellaria paradoxa par tous les paysans est liée beaucoup plus à sa disponibilité et à son accessibilité qu'à sa performance dans l'amélioration des rendements. Mais Cissé et al. (2018), dans leurs travaux sur la perception d'espèces agroforestières et de leurs services écosystémiques par trois groupes ethniques en zone soudanienne du Burkina Faso, ont indiqué que le groupe ethnique, le genre, la taille du ménage, le niveau d'éducation et l'expérience du chef d'exploitation sont des facteurs qui influencent la perception des paysans sur les services écosystémiques des espèces agroforestières.

\section{Effets de la litière foliaire de Vitellaria paradoxa sur le développement du sorgho}

Hauteur à maturité et rendements en biomasses grain et paille du sorgho. $\mathrm{La}$ hauteur du sorgho a été plus élevée $(3,8 \mathrm{~m})$ dans la parcelle ayant reçu la litière pendant trois ans successifs comparativement à celles dans la parcelle traitée pour la première fois avec cette litière $(2,8 \mathrm{~m})$ et le témoin n'ayant jamais reçu cette litière $(3,2 \mathrm{~m})$. Il y a une différence significative $(\mathrm{P}<0,05)$ entre la hauteur du sorgho dans les trois niveaux de traitements (Tableau 5). De même, le traitement "Parcelle de trois ans" a donné les meilleurs rendements en grains et en paille (2081 $\mathrm{kg} \mathrm{ha}^{-1}$ et $4056 \mathrm{~kg} \mathrm{ha}^{-1}$ ), suivi du traitement "Témoin" (703 kg ha" et $2222 \mathrm{~kg}$ ha $^{-1}$ ) tandis que le traitement "Parcelle d'un an" a donné les rendements les moins élevés (353 $\mathrm{kg} \mathrm{ha}^{-1}$ et $1472 \mathrm{~kg} \mathrm{ha}^{-1}$ ). L'analyse statistique a révélé des différences significatives $(P<0,05)$ entre les rendements dans les trois parcelles (Tableau 5).

$\mathrm{Au}$ bout de trois années d'apports successifs, la litière foliaire de Vitellaria paradoxa a favorisé un bon développement végétatif du sorgho et a permis d'avoir les

TABLEAU 4. Fréquences de citation (\%) de l'effet des litières foliaires sur les rendements du sorgho, par espèce et par village

\begin{tabular}{lcccccc}
\hline Espèces & \multicolumn{5}{c}{ Fréquences de citation } & \multirow{2}{*}{ Moyennes } \\
\cline { 2 - 6 } & Louda & Boré & Nakourtenga & Saab-Toécé & Santèna \\
\hline Vitellaria paradoxa & 80 & 49 & 60 & 55 & 52 & 59,2 \\
Anogeissus leiocarpa & 75 & 80 & 92 & 89 & 78 & 82,8 \\
Diospyros mespiliformis & 59 & 66 & 73 & 68 & 50 & 63,2 \\
Combretum micranthum & 85 & 78 & 75 & 69 & 71 & 75,6 \\
Lannea microcarpa & 68 & 76 & 90 & 55 & 65 & 70,8 \\
\hline
\end{tabular}


TABLEAU 5. Hauteurs à maturité $(\mathrm{m})$ et rendements $\left(\mathrm{kg} \mathrm{ha}^{-1}\right)$ en biomasses grain et paille du sorgho dans les traitements (moyennes \pm Ecart type)

\begin{tabular}{llll}
\hline Traitements & Hauteurs & \multicolumn{2}{c}{ Rendements } \\
\cline { 3 - 4 } & & Grain & Paille \\
\hline Témoin & $3,2 \pm 0,35 \mathrm{~b}$ & $703 \pm 107 \mathrm{~b}$ & $2222 \pm 603 \mathrm{~b}$ \\
Parcelle d'un an & $2,8 \pm 0,43 \mathrm{c}$ & $353 \pm 180 \mathrm{c}$ & $1472 \pm 542 \mathrm{c}$ \\
Parcelle de trois ans & $3,8 \pm 0,64 \mathrm{a}$ & $2081 \pm 1215 \mathrm{a}$ & $4056 \pm 1410 \mathrm{a}$ \\
\hline
\end{tabular}

Les moyennes, dans la même colonne, suivies de lettres différentes sont significativement différentes au seuil de 5\% (Test de Fischer)

hauteurs les plus élevées et les meilleurs rendements en grains et en paille. Ainsi, des augmentations de rendement en biomasse atteignant $195 \%$ comparativement au témoin ont été relevées. Ces résultats sont en accord avec ceux de Bado et al. (1997), dans l'Ouest du Burkina Faso, qui ont montré qu'en milieu tropical, les effets des fumures organiques sur l'amélioration des rendements des céréales et du coton devenaient de plus en plus marqués avec le temps. En effet, cette litière foliaire, riche en carbone (Bambara, 2016), constitue un support trophique des organismes du sol tels que les termites, les vers de terre, les fourmis, etc. Le milieu, ainsi traité, devient favorable à leur développement. Ces organismes, particulièrement les termites et les vers de terre souvent appelés « ingénieurs de l'écosystème », en plus de leur rôle dans l'amélioration des propriétés physiques, interviennent dans la fragmentation de la litière et par conséquent la minéralisation du carbone (Lepage, 1981). Cette minéralisation de la matière organique libère, au fil des années, des nutriments indispensables à la croissance des cultures (Zougmoré et al., 2003). L'activité microbienne et l'humidité du sol y sont aussi améliorées (Asimi et al., 2000). Ainsi, le sorgho trouve dans cette parcelle plus d'eau et plus d'éléments nutritifs nécessaires pour son développement et la synthèse de la matière sèche. En cas d'apport de litière sur le long terme, l'influence positive de ces apports sur la production de biomasse résulterait de deux actions différentes. D'une part, elle agirait comme un engrais en fournissant à la plante des éléments nutritifs et, d'autre part, comme amendement en améliorant de nombreuses propriétés physiques et biologiques des sols notamment leur capacité de rétention en eau (Hernando et al., 1989). En milieu tropical notamment, Ganry et al. (2001) ont montré le rôle positif de l'entretien organique des sols, à long terme, dans l'amélioration des rendements des cultures. Mais, le témoin, sans apport de litière, a donné un rendement plus élevé que celui de la parcelle ayant reçu la litière pour la première fois. La minéralisation de la litière, pour libérer des nutriments pour la plante, a probablement été insignifiante, la première année. Par ailleurs, les sols de cette zone étant globalement pauvre en azote (Bado et al., 1997), un apport de litière riche en carbone pourrait entraîner une baisse de rendement des cultures, car le peu d'azote du sol est temporairement utilisé par les microorganismes pour leur métabolisme, créant ainsi le phénomène de "faim d'azote", défavorable au développement du sorgho (Bado et al., 1997). Landry et al. (2013) ajoutent que la litière foliaire qui a un pouvoir absorbant, peut, en première année, réaliser une compétition avec la plante pour l'eau disponible. Ce déficit hydrique peut se traduire par un mauvais développement des cultures. N'Dayegamiye et al. (2005) ont indiqué que l'azote de la litière est principalement sous forme organique et qu'en première année d'application au sol, sa 


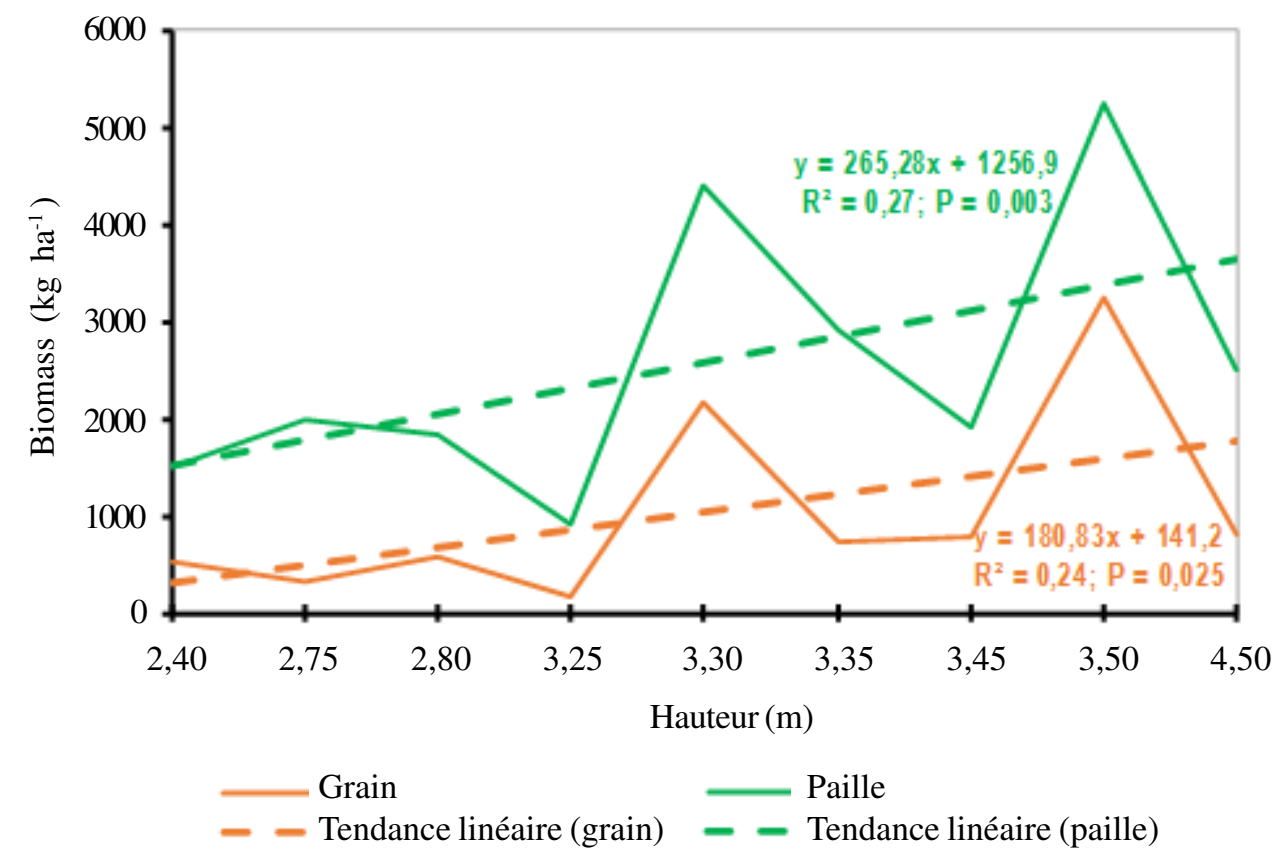

Figure 3. Relation entre les rendements en biomasses et la hauteur du sorgho.

minéralisation pourrait ne pas être suffisante pour combler les besoins en azote des cultures en général.

Relation entre rendements et hauteur de plante. Il existe une corrélation significative $(P<0,05)$ entre les rendements en grains et en paille du sorgho et la hauteur des tiges à maturité (Fig. 3). Les valeurs des coefficients de détermination $\left(\mathrm{R}^{2}\right)$ sont de 0,24 pour les grains et de 0,27 pour la paille, ce qui signifie que les variations de hauteurs expliquent $21 \%$ des variations de rendements en grain et $27 \%$ de celles des rendements en biomasse paille. La corrélation n'est pas la causalité, autrement dit, la grandeur de la taille n'est pas le seul facteur qui explique l'importance du volume des rendements du sorgho.

Les corrélations, entre les rendements et la hauteur du sorgho à maturité, corroborent l'observation faite par Zangré (2000) qui a relevé que, de façon générale, il y a une bonne corrélation entre la croissance de l'appareil végétatif et le rendement céréalier et que les faibles rendements sont dus à une faible croissance de l'appareil végétatif pendant la période semis-floraison.

Les mesures biométriques n'ont été faites que dans les champs traités avec la litière de Vitellaria paradoxa. En perspective, elles devront s'étendre aux parcelles fertilisées avec les litières foliaires de Anogeissus leiocarpa, Combretum micranthum, Lannea microcarpa, Diospyros mespiliformis. Aussi, une étude scientifique sur les caractéristiques chimiques des litières foliaires valorisées et sur leur influence sur les paramètres de fertilité du sol, est à envisager. Les études réalisées en milieu paysan ont l'inconvénient de compter sur la mémoire et l'authenticité des déclarations des interlocuteurs, néanmoins, la démarche adoptée a permis d'obtenir des résultats rationnels.

\section{REMERCIEMENTS}

Ce travail a été rendu possible grâce à un appui financier du projet SOFIIA (Soils of Forest Islands in Africa). Nos remerciements vont à l'endroit des paysans des villages de Louda, 
Boré, Nakourtenga, Saab-Toécé et Santèna pour leur collaboration. M. Ouédraogo Mahamoudou et M. Kirakoya Salifou nous ont accompagnés sur le terrain, nous leur disons merci.

\section{RÉFÉRENCES BIBLIOGRAPHIQUES}

Addinsoft, 2018. XLSTAT statistical and data analysis solution. Paris, France. https:// www.xlstat.com.

Asimi, S., Assa, A., Sedogo, M.P. et Lompo, F. 2000. Influence des modes de gestion des terres sur la respiration du sol et le carbone de la biomasse microbienne. Revue Sciences et Techniques, Sciences Naturelles et Agronomie 24(1):42-53.

Bado, B.V., Sedogo, M.P., Cescas, M.P., Lompo, F. et Bationo, A. 1997. Effet à long terme des fumures sur le sol et les rendements du maïs au Burkina Faso. Cahiers Agricultures 6(6):571-575.

Bambara, D. 2016. Changements climatiques en zones Centre et Nord du Burkina Faso : comparaison entre savoirs locaux et connaissances scientifiques, adaptation par les composts. Thèse de doctorat Univ. Ouagadougou, Burkina Faso. 176pp.

Bambara, D., Ouédraogo, D.B., Nacro, H.B. et Sedogo, M.P. 2017. Evaluation de la productivité céréalière des sols en zone semi-rurale : cas des villages de la zone du projet aéroportuaire de Donsin, Burkina Faso, Afrique Science 13(5):197-209. http:/ /www.afriquescience.net/numero5 _vol_13.html\#.

Bationo, B.A., Kalinganire, A. et Bayala, J. 2012. Potentialités des ligneux dans la pratique de l'agriculture de conservation dans les zones arides et semiarides de l'Afrique de l'Ouest : Aperçu de quelques systèmes candidats. ICRAF Technical Manual n 17 , Nairobi, Kenya. 50pp.

Bayala, J., Balesdent, J., Marol, C., Zapata, F., Teklehaimanot, Z. et Ouédraogo, S.J. 2006. Relative contribution of trees and crops to soil carbon content in a parkland system in Burkina Faso using variations in natural ${ }^{13} \mathrm{C}$ abundance. Journal Nutrient Cycling in Agroecosystems 76:193-201.

Belem, B., Olsen, C.S., Theilade, I., Bellefontaine, R., Guinko, S., Lykke, A.M., Diallo, A. et Boussim, J.I. 2008. Identification des arbres hors forêt préférés des populations du Sanmatenga (Burkina Faso). Bois et Forêts des Tropiques 298(4): 53-64.

Base nationale de données topographiques (BNDT), 2012. Base nationale de données topographiques numériques à référence spatiale. IGB, Ouagadougou, Burkina Faso.

Bognounou, O. 1987. Importance socioéconomique des essences locales de la Haute-Volta: leur importance dans l'alimentation en pays mossi. Notes et documents voltaïques 11(3-4):82-91.

BUNASOLS, 1987. Répertoire des études pédologiques. Doc. Tech., Ouagadougou. 88pp.

Cissé, M., Bationo, B.A., Traoré, S. et Boussim, I.J. 2018. Perception d'espèces agroforestières et de leurs services écosystémiques par trois groupes ethniques du bassin versant de Boura, zone soudanienne du Burkina Faso. Bois et Forêts des Tropiques 338:29-42. DOI : https://doi.org/10.19182/bft2018.338. a31680.

FAO. 2010. Évaluation des ressources forestières mondiales : Rapport national Burkina Faso. FAO, FRA2010/032, Rome. 70pp.

Floret, C. et Pontanier, R. 1997. Amélioration et gestion de la jachère en Afrique de l'Ouest. Actes de l'Atelier Jachère et maintien de la fertilité. IER-ORSTOM, Mali. $153 \mathrm{pp}$.

Fontès, J. et Guinko, S. 1995. Carte de la végétation et de l'occupation du sol du Burkina Faso. Note explicative. Ministère de la coopération française, Projet Campus 88313 101. 67pp.

Ganaba, S., Ouadba, J.M. et Bognounou, O. 1998. Les ligneux à usage de bois d'énergie 
en région sahélienne du Burkina Faso : préférences des groupes ethniques. Sécheresse 9:261-268.

Ganry, F., Feller, C., Harmand, J.M. and Guibert, H. 2001. Management of soil organic matter in semiarid Africa for annual cropping systems. Earth and Environment Sciences 61(1-2):105-118.

Hernando, S., Lobo, M.C. and Polo, A. 1989. Effect of application of a municipal refuse compost on the physical and chemical properties of a soil. Sciences Total Environment 81/82:589-596. DOI: 10.1016/0048-9697(89)90167-8.

Hien, E., Kaboré, W.T., Masse, D. and Dugué, P. 2010. Sustainable Farming Systems in the Sub-Sahelian Zone of Burkina Faso Key Factors. Sustentabilidade em Debate 1(2):126-140.

Jouve, P. 2010. Pratiques et stratégies d'adaptation des agriculteurs aux aléas climatiques en Afrique subsaharienne. S'adapter aux aléas, oui mais comment? Grain de sel 49:15-16.

Kabré, G.W. 2010. Des rameaux ligneux pour fertiliser les sols de savane : quelle disponibilité de la ressource dans le terroir villageois de Guié au Burkina Faso. Mémoire de fin de cycle, ENEF, Bobo-Dioulasso, Burkina Faso. 56pp.

Kessler, J.J. et Boni, J. 1991. Agroforesterie au Burkina Faso bilan et analyse de la situation actuelle. MET DFR - Université Agronomique de Wageningen, Tropical Resource Management Paper No.1, Ouagadougou, Burkina Faso. 144pp.

Landry, C., Boivin, C., Belzile, L. et Mainguy, J. 2013. Essais sur l'apport de différents types de matières organiques appliquées en bande à l'implantation d'un verger de cassis. IRDA, Canada. 50pp.

Lebrun, J.P., Toutain, B., Gaston, A. et Boudet, G. 1991. Catalogue des Plantes Vasculaires du Burkina Faso. IEMVT, CIRAD, France. $341 \mathrm{pp}$.

Lepage, M.G. 1981. L'impact de populations récoltantes de Macrotermes michaeni
(Sjostedt) (Isoptera : Macrotermitinae) dans un écosystème semi-aride (Kajiado, Kenya). Insectes sociaux 28:297-308.

Magioglou, T. 2008. L'entretien non directif comme modèle générique d'interactions. Les Cahiers Internationaux de Psychologie Sociale 78(2):51-65.

MEF, 2010. Profil des régions du Burkina Faso. MEF, Ouagadougou, Burkina Faso. 456pp.

N'Dayegamiye, A., Drapeau, A. et Laverdière, M.R. 2005. Effets des apports de composts de résidus ménagers sur les rendements des cultures et certaines propriétés du sol. Agrosol. 16(2):135-144. Norusis, M.J. 2002. SPSS 11.0. Guide to data analysis, Prentice and Hall. New York, USA.

Ouédraogo, Z.M. 2014. Effets de la couverture du sol à base de Piliostigma reticulatum (De) Hoscht sur l'association sorgho-niébé dans le village de Yilou, Province du Bam (Burkina Faso). Mémoire de Master en production végétale, IDR, Bobo-Dioulasso, Burkina Faso. 65pp.

Reddy, K.C., Berrada, A. et Bonkoula, A. 1998. Manuel d'expérimentation en plein champ à l'usage des cadres de développement agricole. INRAN, Niger. 157pp.

SP/CONEDD, 2006. Programme d'Action National d'Adaptation à la variabilité et aux changements climatiques (PANA). MECV, Ouagadougou. 76pp.

Traoré, L., Ouédraogo, I., Ouédraogo, A. et Thiombiano, A. 2011. Perceptions, usages et vulnérabilité des ressources végétales ligneuses dans le Sud-Ouest du Burkina Faso. International Journal of Biological and Chemical Sciences 5(1):258-278.

Yaméogo, G., Nikiéma, P.P., Yélémou, B., Boussim, J. et Traoré, D. 2005, La gestion de la diversité arborée du parc agroforestier du terroir de Vipalogo, dans le plateau central du Burkina Faso. Cameroon Journal of Experimental Biology 1(2):87-101.

Yélémou, B., Bationo, B., Yaméogo, G. et Millogo-Rasolodimby, J. 2007, Gestion traditionnelle et usage de Piliostigma 
reticulatum (D.C.) Hochst., dans le Plateau central du Burkina Faso. Bois et Forêts des Tropiques 291(1):55-65.

Yélémou, B., Yaméogo, G. et Bationo, B.A. 2013. Le Piliostigma reticulatum au Burkina Faso : Une espèce qui sort de l'ombre. Sahel Agroforesterie 18:6-7.

Zangré, B.V.C.A. 2000. Effets combinés du travail du sol et des amendements organiques sur la fertilité d'un sol ferrugineux lessivé dans la région de Saria (zone centre du Burkina Faso). Mémoire IDR. 83pp.

Zougmoré, R., Zida, Z. and Kambou, N.F. 2003. Role of nutrient amendments in the success of half-moon soil and water conservation practice in semiarid Burkina Faso. Soil and Tillage Research 71:143149. 